\title{
The RFI Environment at Hat Creek Radio Observatory
}

\section{Peter K. G. Williams*}

Dept. of Astronomy, UC Berkeley

E-mail: pwilliamseastro.berkeley.edu

Hat Creek Radio Observatory (HCRO) is the site of the Allen Telescope Array (ATA), a "large number of small dishes" (LNSD) interferometer with continuous frequency coverage between 0.5 and $11 \mathrm{GHz}$. The RFI environment of HCRO is assessed across a wide range of frequencies using ATA science observations. There is significant interference at frequencies below $2.5 \mathrm{GHz}$ and in $\mathrm{C}$ band, though in almost all cases excision techniques suffice to make the data usable. A large fraction of the interference is likely self-generated. A powerful data visualization and editing tool developed for the ATA has revealed broadband RFI emission at frequencies $\lesssim 3 \mathrm{GHz}$, the nature of which is not currently known. Work is ongoing to eliminate self-generated RFI and to understand the nature of the broadband emission.

RFI mitigation workshop - RFI2010,

March 29-31, 2010

Groningen, the Netherlands

${ }^{*}$ Speaker. 


\section{Introduction}

The Hat Creek Radio Observatory (HCRO) is located in northern California, USA and operated by the Radio Astronomy Laboratory (RAL), an organized research unit of the University of California, Berkeley. Since its founding in 1959 the RAL has hosted a variety of telescopes, including an 85-foot (26-meter) single dish and the BIMA ${ }^{1}$ millimeter-wave array. It is currently the site of the ATA, which is a joint project between the RAL and the SETI Institute ${ }^{2}$.

The ATA is a next-generation radio telescope. Its design was based on the recognition that relatively new technologies - most notably, fast and cheap digital hardware-enable the construction of a new kind of radio instrument: a widefield, panchromatic radio camera. For a thorough discussion of the overall ATA design and implementation, see [6]. At the time of writing, the ATA consisted of forty-two 6.1-meter antennas; the complete array is to be composed of 350 elements.

While HCRO is in a relatively remote location amid mountainous terrain, it is not contained within a radio-quiet zone, and there is a nonnegligible and growing amount of human activity near it. Even if this were not the case, emission from satellites is also now virtually inescapable, so an understanding of the RFI environment at HCRO is vital to ATA operations.

\section{Measurements of RFI at HCRO}

In late 2008, extensive observations were made with the ATA covering nearly the entire frequency range between 0.5 and $7 \mathrm{GHz}$ as part of an early science project [7]. An intended side effect of these observations was the creation of a dataset that could be used to assess the RFI environment of HCRO across most of the ATA band.

At the time of these observations, the ATA was equipped with one correlator of $104 \mathrm{MHz}$ bandwidth. Bright calibrator sources were observed at frequencies at multiples of $100 \mathrm{MHz}(1.0 \mathrm{GHz}$, $1.1 \mathrm{GHz}, \ldots)$ for short times $(\sim 90 \mathrm{~s})$. To assess the RFI spectrum, the channelized visibility amplitudes were time-averaged over all observations during a given observing session, regardless of pointing, polarization product, and so on. This approach is obviously reductive, most notably because it is insensitive to low-duty-cycle interferers. However, it does provide a good first-order guide as to which portions of the ATA band are most significantly affected by RFI. RFI spectra were computed for the late 2008 data and augmented with similar data taken in March 2010. The spectra were averaged onto a single grid of 64501 channels ranging from 0.4475 to $7.0524 \mathrm{GHz}$. The median channel had 1430 s of integration contributing to its RFI assessment, though we emphasize that this integration was split between epochs separated by more than a year in some cases.

Figure 1 presents a processed version of this spectrum. (The raw dataset is available upon request.) Gaps have been filled with linear interpolation. A threshold has been applied to define RFI-affected channels, and the spectral occupancy and usable fraction of the correlator bandwidth have been computed in 53-channel bins. RFI primarily affects frequencies $\lesssim 2.5 \mathrm{GHz}$ and $\mathrm{C}$ band, which is used for satellite downlinks and is virtually useless for radio astronomical observations with the ATA. The most significant interferer by far is Sirius/XM satellite radio at $\sim 2.33 \mathrm{GHz}$. The first harmonic of this signal can be seen in Figure 1 at $4.66 \mathrm{GHz}$. Contributions from GPS (1.228,

\footnotetext{
${ }^{1}$ Berkeley-Illinois-Maryland Association; http: / / bima. astro. umd.edu /

${ }^{2}$ Search for Extraterrestrial Intelligence; http: //www. seti.org/
} 


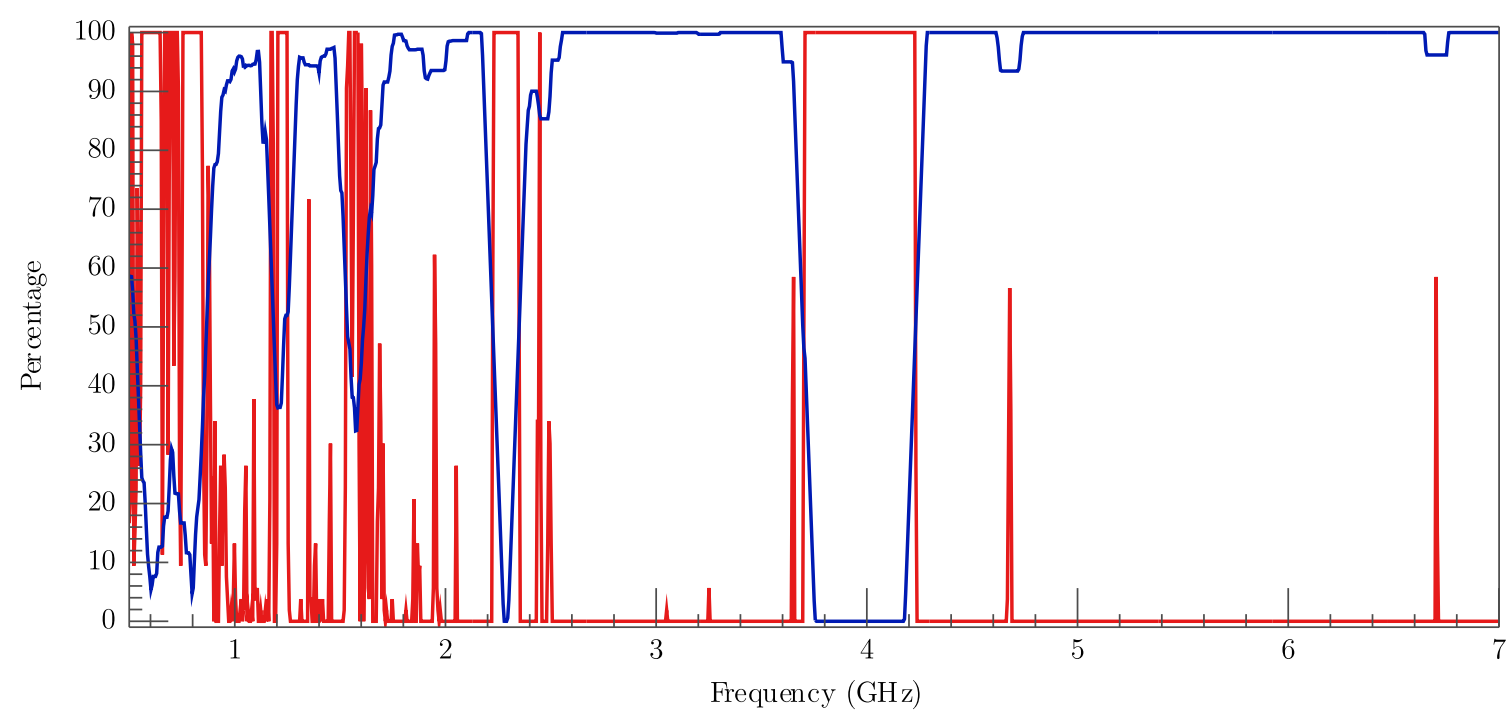

Figure 1: High-duty-cycle RFI spectrum at HCRO in late 2008. Red lines show the spectral occupancy of RFI in $\sim 5-\mathrm{MHz}$ bins of 53 channels each. Blue lines show the usable fraction of the 104-MHz ATA bandwidth if it were centered at each frequency. See the text for discussion, including identifications of the chief interferers.

$1.575 \mathrm{GHz})$, Iridium (1.62 GHz), GLONASS (1.61 GHz), aircraft distance measuring equipment (DME; frequencies between 0.96 and $1.21 \mathrm{GHz}$ ), and terrestial television $(<0.89 \mathrm{GHz})$ are also easily picked out in the full-resolution spectrum. The signal at $\sim 6.7 \mathrm{GHz}$ is unidentified.

It is certain that HCRO self-generates a large amount of RFI. Figure 2 shows measurements made before and after the shutdown of the BIMA array in 2004, when it was relocated to establish CARMA $^{3}$. The measurements were performed with a simple monitor consisting of a discone antenna feeding an off-the-shelf amplifier and digitizer stage. Because BIMA was a millimeter-wave array, HCRO operations were not attuned to the needs of RFI mitigation: most notably, HCRO buildings, including those containing the signal processing hardware, are virtually unshielded and located in the midst of the array. The arrival of the ATA with its large amount of digital hardwarewhich is currently housed in the existing buildings-has almost certainly resulted in even more self-interference. New protocols and infrastructure to deal with RFI are still being established; HCRO equipment and the surrounding region are still not monitored for spurious emissions. Ongoing work to remedy this situation is described in $\S 4$.

\section{A Tool for Data Visualization and Editing}

With its large number of spectral channels and baselines, the ATA data rate is quite large. Traditional hand-editing of data to excise RFI rapidly becomes impractical when working with more than a few hours of ATA data. To address this problem, we have created an interactive tool for visualizing and editing moderate quantities of $u-v$ data. This tool is not a complete solution: datasets comprising hundreds or thousands of hours of ATA observations are simply too large to be tackled in a manual way, and the baseline-oriented approach that its design takes will become

\footnotetext{
${ }^{3}$ Combined Array for Research in Millimeter Astronomy; http: //mmarray . org/
} 


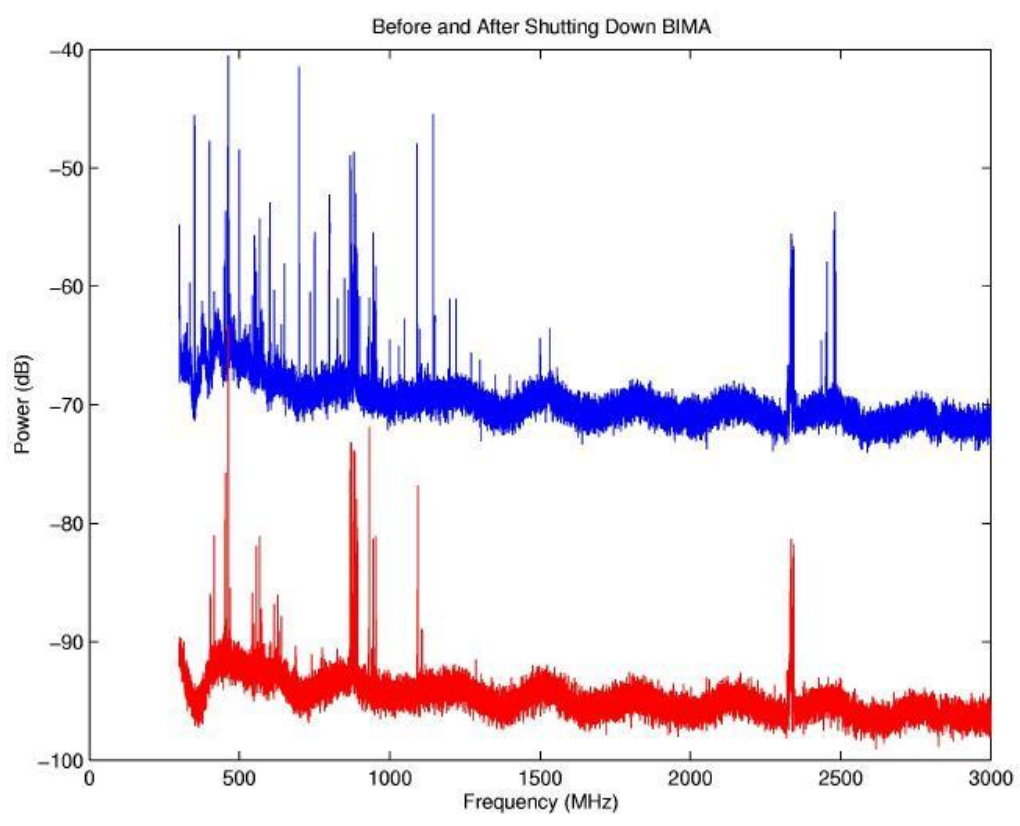

Figure 2: RFI environment at HCRO in 2004. Blue lines show the RFI spectrum while the BIMA array was in operation. Red lines show the spectrum after BIMA was shut down for relocation, with an offset added for clarity. (Courtesy G. C. Bower.)

infeasible with a 350-element array. However, the program has proved to be very helpful for understanding and editing many datasets, and it has revealed an as-yet-undiagnosed broadband interference effect in some observations.

Figure 3 shows a screenshot of the main window of the application. The user is presented with the visibilities for one baseline, represented as a grid in frequency (the horizontal axis) and time (the vertical axis). The user can point and click to select regions to be flagged out from the data. The data can be filtered, sorted, and redisplayed via controls to the left of the screen. Flagged regions can be quickly copied from one baseline to others.

The general structure of this user interface (UI) is nothing new. There are two key aspects to the design of the editor that motivated its creation. Firstly, speed. It cannot be overemphasized how important a responsive UI and efficient command input are to the success of a program such as this one. Switching between baselines in the UI is nearly instantaneous, as is the application of flags from one baseline to another, and the time taken by these operations is nearly independent of data volume. Commands are bound to single keystrokes carefully chosen to minimize the amount of hand motion needed during normal operation. Details such as these make a tremendous difference in the subjective experience of editing a large dataset, and we are convinced that the improved subjective experience leads to better flagging and hence improved quantitative results.

Secondly, even though the program is interactive, it is designed to be used as part of a pipeline. Rather than simply editing a $u-v$ dataset in place, the editor records flagging commands into a separate data file, which can then be applied to input datasets via a standalone, noninteractive utility. The output data products can thus be destroyed and recreated at will. (As a side effect, an 


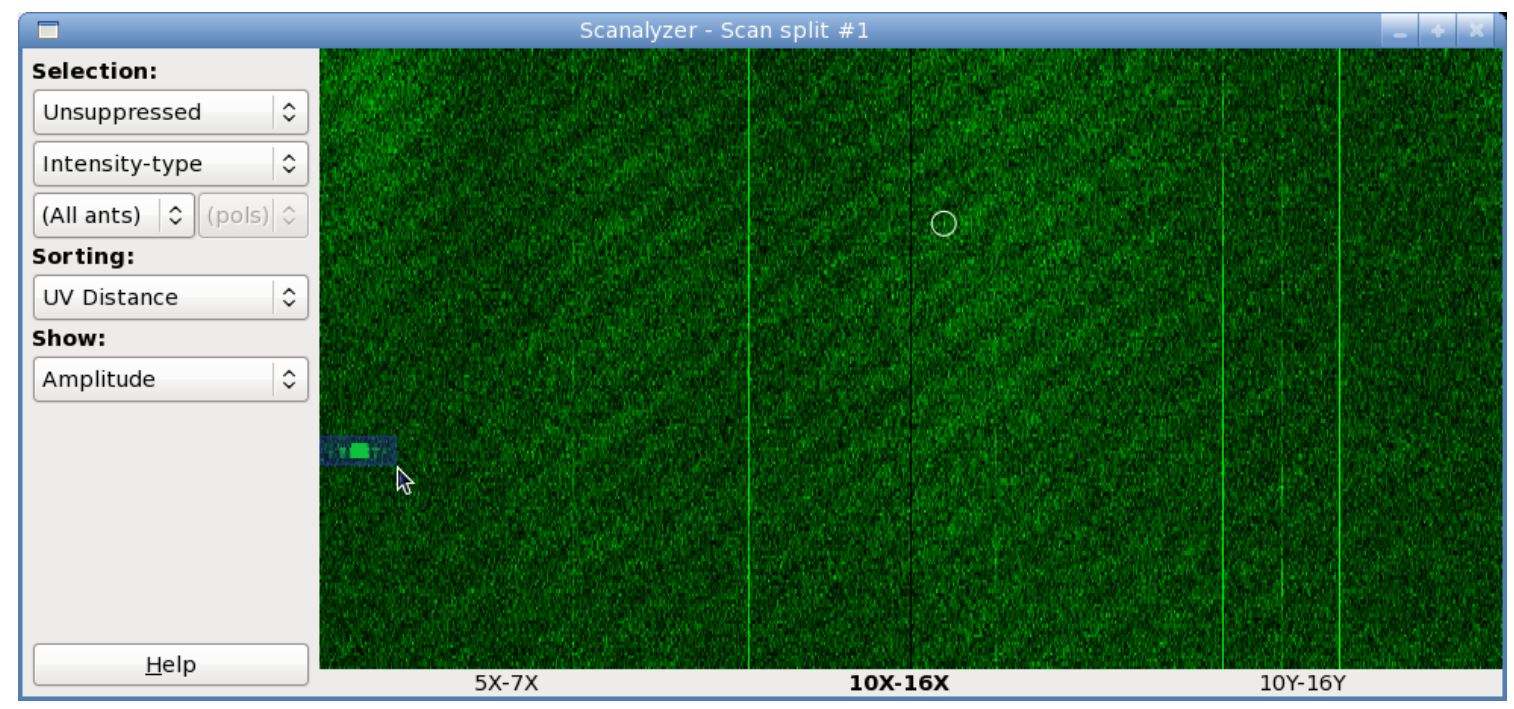

Figure 3: Screenshot typical of the ATA $u$ - $v$ data editor. The center frequency of these data is $1.43 \mathrm{GHz}$. The bandwidth (horizontal axis) spans $104 \mathrm{MHz}$ and the integration time (vertical axis) spans 28 minutes. The $10 \mathrm{X}-16 \mathrm{X}$ baseline is being displayed; $5 \mathrm{X}-7 \mathrm{X}$ and $10 \mathrm{Y}-16 \mathrm{Y}$ are the previous and next baselines, respectively, when ordered by $\sqrt{u^{2}+v^{2}}$. See the text for a brief discussion of the user interface and ripple feature.

exact record of the manual changes to the raw data is maintained.) Furthermore, the flagging data files are small and can be transferred between machines easily, allowing a user to progressively flag a large dataset on a desktop-type machine but process the complete dataset on a server in bulk.

The editor is written in Python and makes heavy use of the GTK+ graphical toolkit and Cairo graphics library. It currently is only suitable for ATA data, but we are eager to make it compatible with any data stored in the MIRIAD data format. Development is ongoing and conducted in an open-source manner, with input and contributions from external developers highly encouraged; the source code is distributed via the Git revision control system ${ }^{4}$.

\subsection{Broadband RFI Features}

There is a notable ripple feature in the data shown in Figure 3. While ATA users had noticed occasional bandpass ripples in plots of ATA visibility spectra, the slow period of the ripple makes it difficult to detect in short integrations, and in many baselines, the ripple is washed out by the noise in an individual spectrum. Visualization of larger datasets in two dimensions (as in Figure 3) makes the effect clear.

Some of these ripples have a fixed periodicity in frequency and time and appear to be artifacts introduced in the ATA signal path. In many observations, however, the ripples precisely track the phase change introduced by fringe rotation and delay correction in the ATA correlator, and are hence consistent with an additive signal in the topocentric frame-that is, they behave like RFI. Unlike typical RFI signals, however, the ripples are broadband. Their prevalence generally seems to increase on shorter baselines and at lower observing frequencies, such that they are largely insignificant in observations made at frequencies above $3 \mathrm{GHz}$.

\footnotetext{
${ }^{4}$ See http://purl.oclc.org/net/pkgwpub/uveditor
} 
Efforts to localize the source via triangulation in the topocentric frame have not yet paid dividends, nor have experiments with all-sky imaging using the AIPY package [5]. A trial application of the RfiX algorithm to ATA data, however, suggests that it has potential for removing at least some instances of the inferfering signal [1].

\section{Outlook}

There are several strands of ongoing work regarding RFI at HCRO. The lowest-hanging fruit, by far, is the improvement of on-site RFI mitigation: all of the available evidence indicates that self-interference is a significant issue, and there are simple steps to be taken to improve the situation. The prospect of retrofitting HCRO buildings with RFI shielding is being investigated, and future construction will involve moving the main array computing hardware to a dedicated, shielded underground vault and relocating the operations buildings as far away from the array as possible. Once these basic steps are taken, a more sophisticated regime of monitoring on-site and regional emissions can be put into place. It is hoped that further analysis of the broadband RFI described in $\$ 3.1$ will elucidate its nature and how it might be mitigated if not eliminated outright. A longer-term goal is to make use of the ATA's flexible hardware and software to test and deploy more sophisticated RFI mitigation schemes. As an example, work is underway to investigate the use of some ATA FPGAs to monitor spectral kurtosis in a realtime RFI excision system [4]. The unique capabilities of the ATA give it unique potential as a testbed for such advanced mitigation techniques.

\section{References}

[1] R. Athreya, A New Approach to Mitigation of Radio Frequency Interference in Interferometric Data, ApJ, 696, 885 (2009) [arXiv: 0902 .3332].

[2] C. Barnbaum, R. F. Bradley, A New Approach to Interference Excision in Radio Astronomy: Real-Time Adaptive Cancellation, ApJ, 116, 2598 (1998).

[3] A. Leshem, A.-J. van der Veen, A.-J. Boonstra, Multichannel Interference Mitigation Techniques in Radio Astronomy, ApJSS, 131, 355 (2000) [ast ro-ph / 0005259 ].

[4] G. M. Nita, D. E. Gary, Z. Liu, G. J. Hurford, S. M. White, Radio Frequency Interference Excision Using Spectral-Domain Statistics, PASP, 119, 805 (2007).

[5] A. R. Parsons, D. C. Backer, Calibration of Low-Frequency, Wide-Field Radio Interferometers Using Delay/Delay-Rate Filtering, AJ, 138, 219 (2009) [arXiv: 0901.2575$].$

[6] J. Welch, D. Backer, L. Blitz, D. C.-J. Bock, et al., The Allen Telescope Array: The First Widefield, Panchromatic, Snapshot Radio Camera for Radio Astronomy and SETI, Proc. IEEE, 97, 1438 (2009) [arXiv:0904.0762].

[7] P. K. G. Williams, G. C. Bower, Evaluating the Calorimeter Model with Broadband, Continuous Spectra of Starburst Galaxies Observed with the Allen Telescope Array, ApJ, 710, 1462 (2010) [arXiv:0912.0014]. 